\title{
A particle packing algorithm for packed beds with size distribution
}

\author{
G. A. Georgalli · M. A. Reuter
}

Received: 11 December 2006 / Published online: 4 March 2008

(C) The Author(s) 2008

\begin{abstract}
A particle packing algorithm for simulating realistic packed beds of spheres with size distribution is described. The algorithm used the Monte Carlo method combined with the simulated annealing minimisation algorithm to solve the packed bed simulations. The objective function which was minimised was a combination of two functions, one describing the deviation from the target mean coordination number of the spheres in each size interval and the other the average fraction of overlapping volume of the spheres per contact. In this way a realistic bed structure was maintained while at the same time controlling the coordination number of the spheres. The algorithm used an experimentally validated model to predict the mean coordination number of the spheres in each size interval.
\end{abstract}

Keywords Particle packing algorithm .

Simulated annealing $\cdot$ Coordination number

\section{List of symbols}

Arabic symbols

$D_{N} \quad$ neighbouring distance (m)

E simulated annealing energy (-)

$k \quad$ simulated annealing Boltzmann constant (-)

$m \quad$ number of spheres (-)

$P \quad$ Boltzmann probability (-)

\section{G. A. Georgalli $(\bowtie)$}

Department of Materials Science and Engineering,

Technical University of Delft, Mekelweg 2,

2628 CD Delft, The Netherlands

e-mail: g.a.georgalli@gmail.com

\section{A. Reuter}

Department of Chemical and Biomolecular Engineering,

University of Melbourne, Melbourne, VIC 3010, Australia

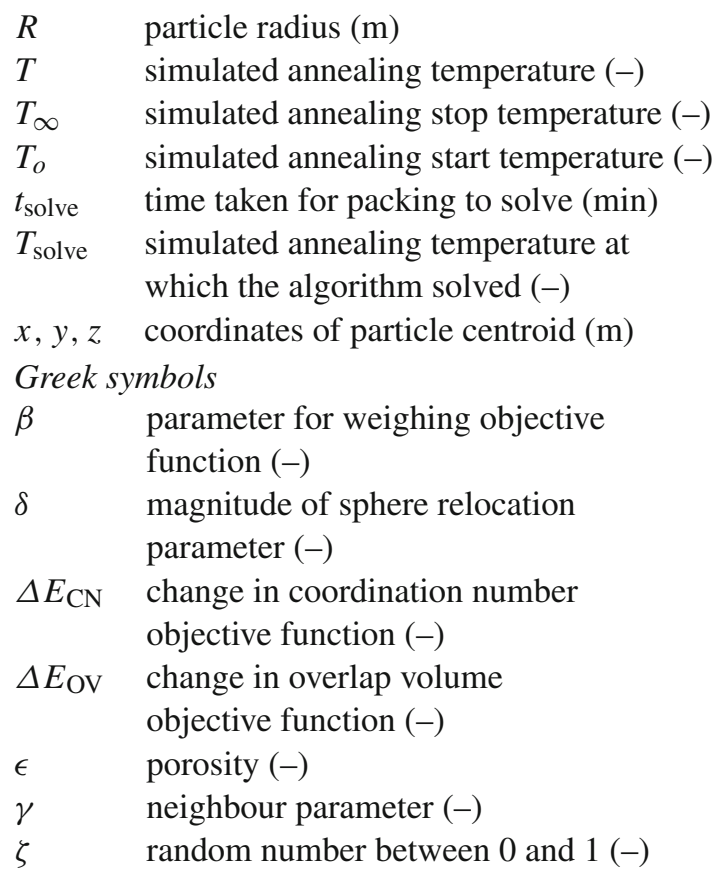

\section{Introduction}

In this paper a packing algorithm which simulates realistic packing structures is proposed. The packing structure is defined by the coordination number of the spheres. The mean coordination number of the spheres within each size interval is determined using a model which is a function of the overall bed porosity and the size distribution of the spheres [1-4]. The model has been experimentally validated using multi-sized glass spheres and a CT scanner [5].

Due to the difficulties associated with the experimental study of packed particulate assemblies, much of the knowledge about packed beds is derived from particle packing 
algorithms. For this reason most particle packing algorithms are created in such a way as to be used to determine certain properties of packed beds such as densest possible packing $[6,7]$ and the associated coordination number, as well as other properties such as the radial distribution of porosity and coordination number $[8,9]$. Often the mechanics of the packing process are part of the packing algorithm and thus it is expected that the outputs are representative of real packed beds.

The algorithm proposed in this paper follows a different path. The mechanics of packing are not used at all, the spheres are simply moved around until the required objectives are met. The algorithm assumes that the packing structure exists and subsequently tries to computationally recreate it. This is made possible by the use of an experimentally validated model to predict the mean coordination number of the spheres in each size interval [5].

Clearly the coordination number cannot be the sole control variable for the algorithm as this would result in spheres lumping together in a physically impossible way. In order to avoid this a second control variable is introduced, the mean overlap volume. By minimising the two functions which describe the deviation from the desired mean coordination number and mean overlap volume of the spheres it is possible to create realistic packing structures.

The packing structures were constructed using the Monte Carlo method. The optimisation was done using the simulated annealing minimisation algorithm [10].

\section{Simulated annealing}

The method of simulated annealing is a technique which is suitable for optimisation problems of very large scale. The method is based on an analogy with thermodynamics, specifically the way the liquids freeze or crystallise, or metals cool and anneal. It is based on the Boltzmann probability distribution [11].

$\operatorname{Prob}(E) \approx \exp \left(\frac{-E}{k \cdot T}\right)$

The equation, when applied to an optimisation problem, is expressed as follows:

$P=\exp \left[\frac{-\left(E_{2}-E_{1}\right)}{k \cdot T}\right]=\exp \left[\frac{-(\Delta E)}{k \cdot T}\right]$

From Eq. 2 we can see that if $E_{2}<E_{1}$ the probability is greater than unity. In these cases the change is assigned a probability of 1 , and so the solution is always accepted. If $E_{2}>E_{1}$ then the value of $P$ is between 0 and 1 . This value is compared to a randomly generated value between 0 and 1 , if the randomly generated value is less than the calculated probability then the solution is accepted, if it is greater then it is not accepted. In summary, we can see that if a solution is better, then it is always selected. If it is worse, it is sometimes selected, with fewer worse configurations accepted as the temperature decreases.

\section{Objective function}

The objective function is comprised of two functions, one which describes the deviation from the target mean Coordination Number of the spheres (the $\mathrm{CN}$ objective function), and another which describes the mean magnitude of Overlapping Volume for each contacting sphere (the OV objective function).

The OV objective function is the average fraction of the spheres volume that is overlapping per contact. The spheres are seen as virtual hard spheres and are allowed to overlap one another throughout the running of the packing algorithm. As the algorithm progresses, the mean OV of the packed bed is minimised to below the target value of $1 \%$ per contact. Clearly this is a departure from real spheres but for an average final intersecting volume of less than $1 \%$ it is seen as a reasonable approximation. It is calculated by dividing the sum of the fractional OV of each sphere by the number of spheres $m$. The fractional OV of each sphere is simply the sum of the volume of said sphere (sphere $i$ ) which is overlapping with it's contacting spheres (sphere $j$ ), divided by the volume of sphere $i$.

As described in the Introduction the target mean $\mathrm{CN}$ of the spheres in each size interval is predicted using an experimentally validated $\mathrm{CN}$ model [1-5]. The average of the CN's of all the spheres in the same size interval should approach the target mean $\mathrm{CN}$ for that size interval during the progression of the algorithm. The CN's of the spheres in the same size interval can therefore have a distribution. The distribution of the CNs within the bed is only limited by the lower CN limit, which is equal to 2 for a packed bed [1]. The $\mathrm{CN}$ objective function consists of two parts which are added together. The first part describes the deviation from the desired mean $\mathrm{CN}$ of the spheres in each size interval. This is equal to the absolute value of the difference between the desired model predicted mean and actual mean $\mathrm{CN}$ of the spheres in the respective intervals. The second part of the $\mathrm{CN}$ objective function levies a penalty to any spheres which have an unrealistic $\mathrm{CN}$ (less than 2). The solving limit of the $\mathrm{CN}$ objective function was also set at $1 \%$.

The objective function minimised by the simulated annealing algorithm when solving the packing problem is a combination of the $\mathrm{CN}$ and $\mathrm{OV}$ objective functions. The way that these two functions are combined to form a single objective function is not as elementary as simply adding them together. The problem relates to the magnitudes of the differences in the objective functions value prior to and after a sphere 
relocation (we shall call this $\Delta E$ ). If, once we make a relocation, the magnitude of $\Delta E$ for one of the objective functions is consistently much greater than that of the other objective function, then the objective function with the consistently larger $\Delta E$ will be favoured to the detriment of the other. In this case the $\mathrm{CN}$ objective function was found to be favoured. The situation described above is avoided by combining the two objectives into a weighted sum as shown in Eq. 3.

$$
\Delta E=\beta \cdot \Delta E_{\mathrm{OV}}+(1-\beta) \cdot \Delta E_{\mathrm{CN}}
$$

The value of $\Delta E$ as shown in Eq. 3 is then used in the simulated annealing equation (Eq. 2).

\section{Algorithm structure}

The particle packing algorithm can be split into two parts, namely packing initiation and packing refinement. The algorithm is shown graphically in Fig. 1. During packing initiation the spheres are randomly located within the packing space. The packing space is defined using the desired porosity for the packing, the total volume of the spheres and the height to diameter ratio of the cylindrical packed bed. The desired porosity as well as the packed bed height to diameter ratio are chosen by the user. For all the packing simulations a height to diameter ratio of 1 was used. The mean $\mathrm{CN}$ target for each interval as well as the initial values of the $\mathrm{CN}$ and OV objective functions are also calculated at this time and the neighbour list of each particle is populated (this will be explained later).
Once the packing is initiated the packing refinement starts. It is worthwhile noting that the packing refinement stage in no way attempts to recreate the physics of particles packing together, as such there are no forces calculated on the spheres. During each iteration a random sphere is selected and its centroid coordinates relocated to randomly generated $x-y-z$ coordinates within a restricted cubic space. The magnitude of the movement is determined by $\delta$ (see Eqs. 4 through 6). Smaller movements make the algorithm minimise quickly, but can also lead to it becoming stuck in local minima. With larger movements the minimisation is slower but more effective.

$$
\begin{aligned}
& x_{i, \text { new }}=x_{i}+\delta \cdot R_{i}(2 \zeta-1) \\
& y_{i, \text { new }}=y_{i}+\delta \cdot R_{i}(2 \zeta-1) \\
& z_{i, \text { new }}=z_{i}+\delta \cdot R_{i}(2 \zeta-1)
\end{aligned}
$$

Once the sphere has been relocated the objective function needs to be recalculated and compared to its value prior to the relocation of the sphere. In order to speed up the calculation of the objective function, only the relocated sphere and the spheres which are said to be neighbouring it will have their $\mathrm{CN}$ and mean $\mathrm{OV}$ re-calculated. In order to speed the algorithm up further, not all of the spheres are assessed when recalculating the objective functions after a relocation. This is achieved by modifying the previous values of the objective functions by only taking into account the changes of the coordination number and mean overlap volume of the relocated sphere and its neighbours, instead of totally recalculating them from scratch using all of the spheres.

\begin{tabular}{|c|c|c|c|c|c|c|c|c|c|c|c|c|c|c|c|}
\hline \multirow[b]{2}{*}{$\epsilon$} & \multicolumn{5}{|c|}{ Log-uniform } & \multicolumn{5}{|c|}{ Rosin-Rammler } & \multicolumn{5}{|c|}{ Andreasen } \\
\hline & $m$ & $\begin{array}{l}\text { OV } \\
(\%)\end{array}$ & $\begin{array}{l}\mathrm{CN} \\
(\%)\end{array}$ & $\begin{array}{l}t_{\text {solve }} \\
(\mathrm{min})\end{array}$ & $T_{\text {solve }}$ & $m$ & $\begin{array}{l}\text { OV } \\
(\%)\end{array}$ & $\begin{array}{l}\mathrm{CN} \\
(\%)\end{array}$ & $\begin{array}{l}t_{\text {solve }} \\
(\mathrm{min})\end{array}$ & $T_{\text {solve }}$ & $m$ & $\begin{array}{l}\text { OV } \\
(\%)\end{array}$ & $\begin{array}{l}\mathrm{CN} \\
(\%)\end{array}$ & $\begin{array}{l}t_{\text {solve }} \\
(\mathrm{min})\end{array}$ & $T_{\text {solve }}$ \\
\hline \multirow[t]{2}{*}{0.3} & 4820 & 0.93 & 0.95 & 449 & 4.25 & 4710 & 1.00 & 0.91 & 309 & 23.08 & 4960 & 1.00 & 0.67 & 333 & 18.80 \\
\hline & 2410 & 0.93 & 0.89 & 291 & 3.29 & 2355 & 0.99 & 0.49 & 187 & 9.65 & 2480 & 1.00 & 0.27 & 159 & 14.55 \\
\hline \multirow[t]{2}{*}{0.4} & 4820 & 0.96 & 0.95 & 205 & 11.26 & 4710 & 1.00 & 0.96 & 139 & 29.83 & 4960 & 0.75 & 0.99 & 203 & 18.8 \\
\hline & 2410 & 0.90 & 0.89 & 114 & 9.17 & 2355 & 0.99 & 0.97 & 56 & 36.63 & 2480 & 0.88 & 0.87 & 75 & 26.92 \\
\hline
\end{tabular}

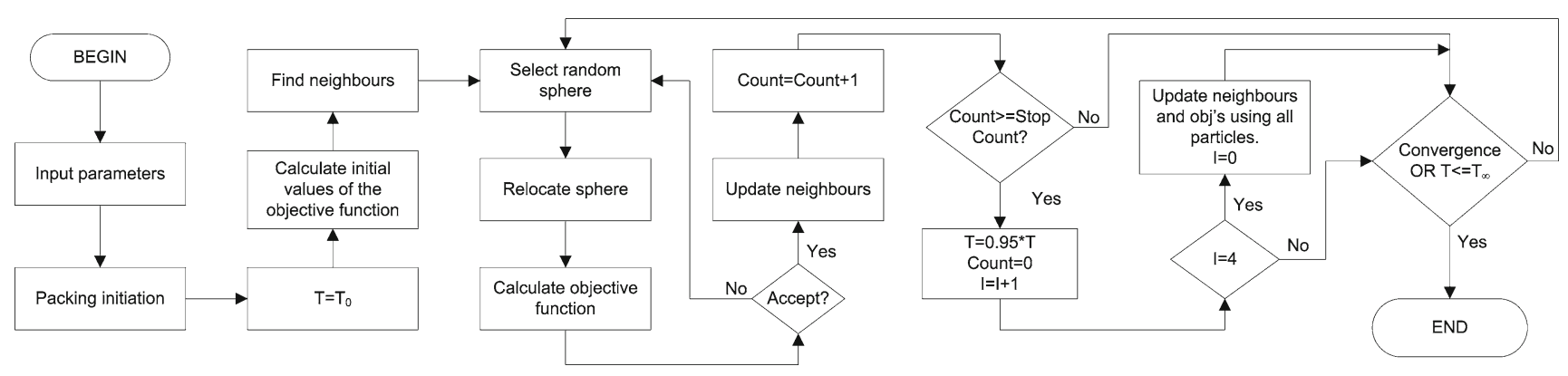

Fig. 1 Flowchart of algorithm structure

Table 1 Relevant results from packing attempts 

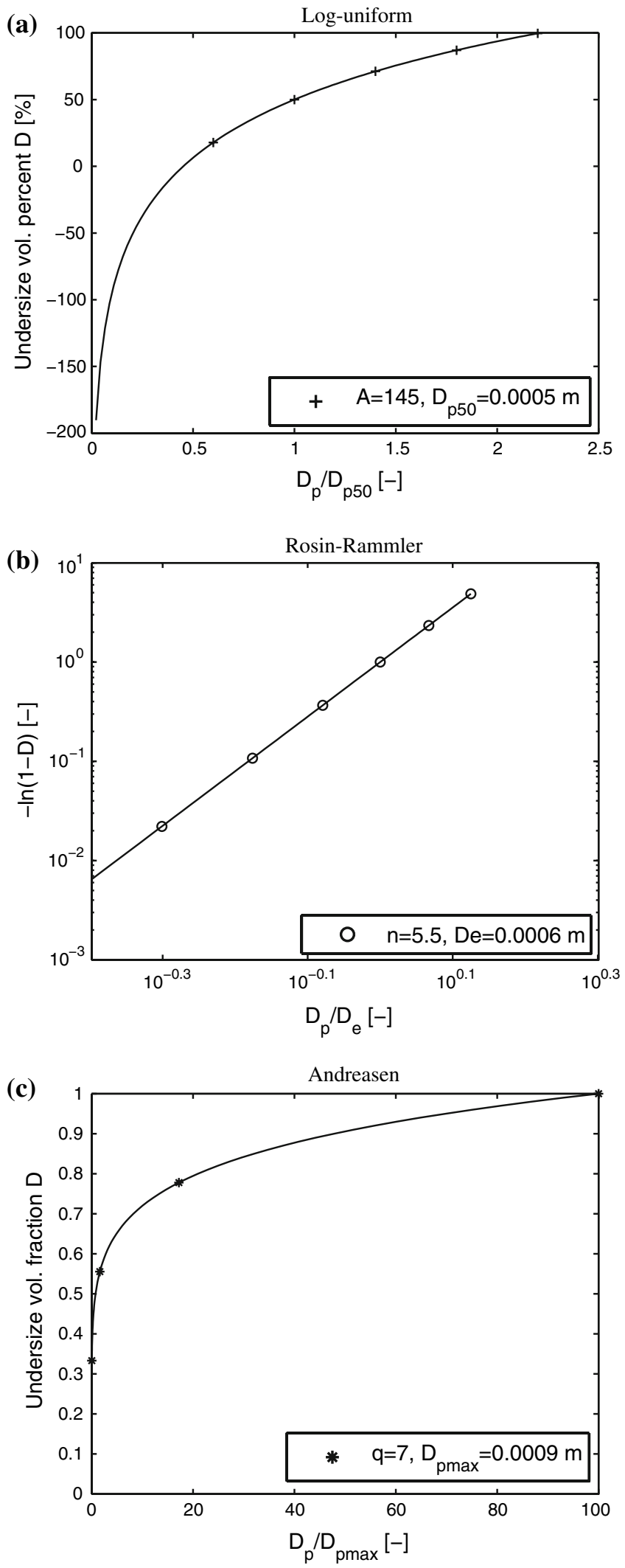

Fig. 2 Cumulative distributions used for packing attempts
$D_{N}=\gamma\left(\sqrt{3 \cdot\left(\delta \cdot R_{i}\right)^{2}}+R_{i}+R_{j}\right)$

A sphere is said to be a neighbour if the Euclidean distance between its centroid and that of the relocated sphere prior to relocation is less than or equal to $D_{N}$ as described in Eq. 7 . Finding the neighbours of all of the spheres in the bed is quite computationally intensive as each sphere must be evaluated relative to every other sphere. The number of calculations increase quadratically relative to the number of spheres in the bed. During packing initiation the neighbours of all of the spheres are identified in this computationally intensive way.

Once a relocation has been made, and the new value of the objective function calculated, the decision on whether to accept the relocation or not is made by applying the simulated annealing criteria [10].

After each accepted sphere relocation the list of neighbours for the relocated sphere needs to be updated. In order to speed up the re-identification of neighbouring spheres the value of $\gamma$ in Eq. 7 is increased to a value greater than 1 and only the relocated sphere, its neighbours, and its neighbours are assessed. This allows one to identify all of the neighbouring spheres without assessing all of the spheres in the bed. The use of the neighbours results in an algorithm where the number of calculations necessary for each relocation (be it accepted or not) is independent of the number of spheres in the bed.

When the number of accepted relocations is equal to 20 times the sphere count, the temperature is decreased. In this application a proportional cooling schedule was used, with the new temperature being set equal to $95 \%$ of the previous temperature. These values were chosen by trial and error and were found to supply the best compromise between the time taken for the algorithm to solve and the ability of the simulated annealing algorithm to minimise the objective function to below the target. If the number of accepted relocations prior to a temperature decrease and the cooling ratio are decreased, then the algorithm will solve faster but may become stuck in a local minimum. The choice of the starting simulated annealing temperature $\left(T_{o}\right)$ and the Boltzmann constant $(k)$ should be such that at the first temperature decrease the number of accepted relocations should be equal to roughly $50 \%$ of the total relocations [10]. In order to ensure that any error which may creep into the calculations due to the use of the neighbours is accounted for, the objective functions as well as the list of neighbours of each sphere are recalculated using all of the spheres in the bed every four temperature steps. The algorithm will continue until both the $\mathrm{CN}$ and $\mathrm{OV}$ objective function values are below their respective targets, or the temperature decreases to below the minimum temperature $\left(T_{\infty}\right)$. 


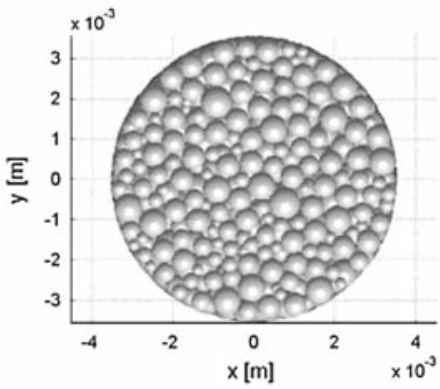

(a) $x-y$ axis

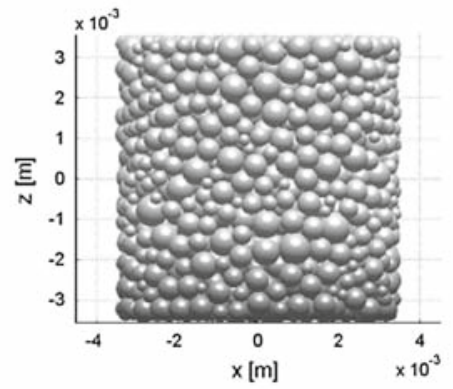

(b) $x-z$ axis

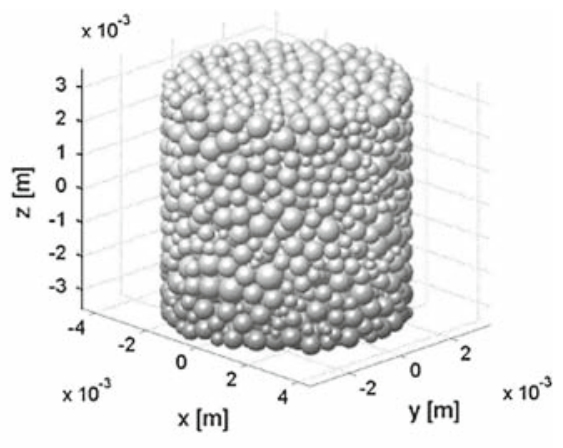

(c) $x-y-z$ axis

Fig. 3 3-D representation of packing for Rosin-Rammler, $m=2355, \epsilon=0.3$ [12]

\section{Results}

All of the pellet design simulations were run on a hp workstation $\mathrm{xw} 8000$ which contained an Intel ${ }^{\circledR} \mathrm{Xeon}^{\mathrm{TM}} 3.06 \mathrm{Ghz}$ processor. The packing problem was solved for three types of size distribution, namely log-uniform, Rosin-Rammler and Andreasen (see Fig. 2). One configuration was solved for each distribution. For each configuration two different porosity $(\epsilon)$ values were used, 0.3 and 0.4 . For each configuration and porosity two simulations were done, with one having twice the number of spheres as the other. The simulations were run from an initial simulated annealing temperature $\left(T_{0}\right)$ of 300 with the end temperature $\left(T_{\infty}\right)$ equal to 0.1 and the Boltzmann constant $k$ equal to $6 \times 10^{-4}$ divided by the number of spheres $m$. The number of accepted iterations in each temperature was equal to 20 times the number of spheres, after which the temperature was proportionally decreased by a factor of 0.95 . The parameters $\delta, \beta$ and $\gamma$ were set at 0.3 , 0.9 and 1.30 respectively. The selection of a value for $\beta$ was done by trial and error. For values lower than 0.9 the $\mathrm{CN}$ objective function was found to be favoured in that it would meet its solving criterion long before the OV objective function. For values higher than 0.9 the $\mathrm{CN}$ objective function would sometimes not meet its solving criteria at all.

From Table 1 it is apparent that the solving time $\left(t_{\text {solve }}\right)$ of the simulations is linear with respect to the number of particles in the bed, that is, for the same size distribution and porosity the beds with double the amount of particles take roughly double the time to solve. This is due to the use of the neighbours, which renders the calculations done per particle relocation independent of the number of particles in the bed.

Sometimes the smaller system solves in less than half the time of the larger system or vice versa. In these situations the solution is simply reached at an earlier or later time in the annealing process, as is evident if one considers the temperature at which the algorithm reached a solution $\left(T_{\text {solve }}\right)$. Even though the different distributions have a similar amount of particles, some solve quicker than others. Typically those which have a large number of particles which require either relatively high CNs (e.g. Log-uniform, $\epsilon=0.3$ where the desired mean $\mathrm{CN}$ of the larger particles is equal to 33) or relatively low CNs (e.g. Andreasen, $\epsilon=0.4$ where the desired mean $\mathrm{CN}$ of the smaller particles is equal to 3 ) take a longer time to solve. With high CNs there are more contacts between particles which can lead to higher OVs between the particles which need to be minimised. With lower CNs the lower $\mathrm{CN}$ limit of 2 restricts the range of available $\mathrm{CNs}$ which can be used to meet the desired mean CN. A 3-D representation of a selected packing output is shown in Fig. 3 .

\section{Conclusions}

The algorithm effectively used the Monte Carlo method combined with the simulated annealing minimisation algorithm to solve the packing simulations using a combination of two objective functions. One describing the deviation from the target $\mathrm{CN}$ of the spheres and the other the average OV per contact of the spheres. The target $\mathrm{CN}$ was obtained using a model which was validated experimentally using a CT scanner, which is unique for particle packing algorithms.

Simulations were successfully completed for a range of size distributions and porosities. By creating and constantly updating a list of neighbouring sphere for each sphere the number of calculations per sphere relocation becomes independent of the number of spheres in the bed. This allows for beds with a large number of spheres to be simulated in a relatively short time.

Generally the algorithm took a longer time to solve systems which had size intervals with either relatively high or relatively low target mean CNs. This was due to the difficulty associated with minimising the $\mathrm{OV}$ objective for a large number of contacts, and the difficulty in meeting low mean $\mathrm{CNs}$ while working with the lower $\mathrm{CN}$ limit of 2 . 
Acknowledgements This work is part of the E.E.T. (Economy, Ecology and Technology) project (http://www.vg2.nl) supported by the Dutch government. The financial support from the E.E.T. program is gratefully acknowledged.

Open Access This article is distributed under the terms of the Creative Commons Attribution Noncommercial License which permits any noncommercial use, distribution, and reproduction in any medium, provided the original author(s) and source are credited.

\section{References}

1. Suzuki, M., Oshima, T.: Estimation of the co-ordination number in a multi-component mixture of spheres. Powder Technol. 35, 159$166(1983)$

2. Suzuki, M., Oshima, T.: Comparison between the computersimulated results and the model for estimating the co-ordination number in a three-component random mixture of spheres. Powder Technol. 43, 19-25 (1985)

3. Suzuki, M., Oshima, T.: Co-ordination number of a multicomponent randomely packed bed of spheres with size distribution. Powder Technol. 44, 213-218 (1985)
4. Gotoh, K.: J. Soc. Pow. Tech. 15, 220 (1978)

5. Georgalli, G.A., Reuter, M.A.: Modelling the co-ordination number of a packed bed of spheres with distributed sizes using a CT scanner. Miner. Eng. In Press (2005)

6. Sobolev, K., Amirjanov, A.: A simulation model of the dense packing of particulate material. Adv. Powder Technol. 15(3), 365376 (2004)

7. Sobolev, K., Amirjanov, A.: The development of a simulation model of the dense packing of large particulate assemblies. Powder Technol. 141, 155-160 (2004)

8. Reyes, S.C., Iglesia, E.: Monte Carlo simulations of structural properties of packed beds. Chem. Eng. Sci. 46(4), 1089-1099 (1991)

9. Abreu, C.R.A., Macias-Salinas, R., Tavares, F.W., Castier, M.: A Monte Carlo simulation of packing and segregation of spheres in cylinders. Braz. J. Chem. Eng. 16(4), 395-405 (1999)

10. Kirkpatrick, S., Gelatt, C.D., Vecchi, M.P.: Optimization by simulated annealing. Science 220, 671-689 (1983)

11. Press, W.H., Flannery, B.P., Teulosky, S.A., Vetterling, W.T.: Numerical Recipes in C. Cambridge University Press, Cambridge (1988)

12. Bodin, P.: Bubbleplot3.m. http://www.mathworks.com (2005) 